\title{
O NÚMERO BÁSICO DE REPRODUÇÃO DE UMA DOENÇA E A MATRIZ PRÓXIMA GERAÇÃO*
}

\author{
MARIA HERMÍNIA DE PAULA LEITE MELLO ${ }^{\dagger}$ \\ $\mathrm{E}$ \\ RÔMULO FERNANDO DOMINGOS DA SILVA
}

\begin{abstract}
Resumo
O principal parâmetro para avaliar a intensidade de transmissão de uma doença infecciosa é o Número Básico de Reprodução da doença, denotado por $\mathrm{R}_{0}$. $\mathrm{O}$ número $\mathrm{R}_{0}$ é um indicador do limiar epidêmico da doença. Sua determinação permite a compreensão da dinâmica da propagação da doença e a definição de estratégias para o controle da mesma. O objetivo desse trabalho é descrever um método algébrico para o cálculo do número $\mathrm{R}_{0}$, que utiliza uma matriz, chamada Matriz Próxima Geração, a qual estará associada a um modelo matemático da doença. Aplicamos o método para a determinação do número $\mathrm{R}_{0}$ em uma modelagem, por meio de equações diferenciais ordinárias, da Leishmaniose Canina.
\end{abstract}

\section{Introdução}

Muitas doenças infecciosas podem ser modeladas usando um modelo de compartimentos. Ao sistema de compartimentos, associamos um sistema de equações diferenciais ordinárias autônomas, que chamamos de sistema autônomo e que será o modelo matemático para a descrição da dinâmica da propagação da doença.

O principal parâmetro para avaliar a intensidade de transmissão da infecção é o Número Básico de Reprodução da Doença, denotado por $\mathrm{R}_{0}$, que é definido como sendo o número de infecções secundárias produzidas por um único indivíduo infectado, em uma população totalmente suscetível. Portanto, esta medida possibilita calcular o impacto de uma infecção através da sua capacidade de repassar a doença para novos indivíduos.

\footnotetext{
* Palavras-chave: Sistemas Autônomos, Modelagem em Epidemiologia, Número Básico de Reprodução de uma doença, Matriz Próxima Geração

†Prof. Associado IME/ UERJ; E-mail: mhermínia@ime.uerj.br

${ }^{\S}$ E-Mail: romulouerj2013@gmail.com
} 
Para cada modelo matemático dado por um sistema autônomo, o Número Básico de Reprodução da Doença será determinado através de uma matriz, chamada Matriz Próxima Geração, conceito este introduzido, em 1990, por Diekmann, Heesterbeek e Metz, que associaram o número $\mathrm{R}_{0}$ ao raio espectral da Matriz Próxima Geração (DIEKMANN et. al., 1990).

Para exemplificar, aplicamos o método da Matriz Próxima Geração para a determinação do número $\mathrm{R}_{0}$ em um sistema autônomo apresentado em Dye (1966), que modela a Leishmaniose Visceral Canina. $\mathrm{O}$ artigo citado exibe o número $\mathrm{R}_{0}$ da Leishmaniose Visceral, associado a um modelo matemático utilizado para descrever a doença, mas não faz referência de como esse número foi obtido. Assim, este trabalho complementa o referido artigo, uma vez que descreve todas as etapas de um método algébrico para determinar o número $\mathrm{R}_{0}$.

\section{Modelagem Matemática em Epidemiologia - Sistema Autônomo}

Em muitos modelos matemáticos aplicados à epidemiologia, a fim de descrever a propagação de doenças infecciosas como dengue, malária e leishmaniose consideramse quatro tipos de população:

- a população formada por indivíduos suscetíveis a doença;

- a população formada por indivíduos infectados, que não são infectantes, pois nesse momento não são capazes de transmitir a doença, chamada de população latente ou de indivíduos expostos;

- a população formada por indivíduos que transmite a doença, população essa denominada de indivíduos infectantes ou infecciosos;

- a população formada por indivíduos recuperados.

As populações são denotadas por $S=S(t), E=E(t), I=I(t)$ e $R=R(t)$, respectivamente, e o modelo de compartimentos é conhecido como modelo SEIR. A população formada por indivíduos expostos ou latentes, também costuma ser denotada por $L=L(t)$.

O modelo matemático, aqui considerado, será dado por um sistema autônomo de equações diferencias, obtido a partir de um sistema de compartimentos do tipo SEIR. O estudo da dinâmica da doença, se esta poderá ser controlada ou se resultará em uma 
epidemia, não pode ser determinado unicamente a partir da análise da estrutura do modelo matemático que descreve a doença. Primeiramente, é necessário fazer uma distinção entre indivíduos infetados e indivíduos não infectados, dada pela interpretação epidemiológica do modelo.

Considere uma população heterogênea de indivíduos, mas cujos indivíduos podem ser agrupados em $n$ compartimentos homogêneos.

Seja $x=x(t)=\left(x_{1}, x_{2}, \ldots, x_{n}\right)$, com cada coordenada $x_{i}=x_{i}(t)$ representando o número de indivíduos do compartimento $i, 1 \leq i \leq n$. Por indicar uma população de indivíduos, $x_{i}=x_{i}(t) \geq 0$, para todo $t \geq 0$. Além disso, a fim de facilitar o entendimento do método e cálculos posteriores, faremos uma ordenação nas coordenadas do vetor $x=x(t)$, considerando as primeiras $m$ coordenadas do vetor, $m<n$, correspondentes às populações de indivíduos infectados. No modelo SEIR, consideramos duas categorias de indivíduos infectados: expostos e infectantes, com a ordenação $x=x(t)=\left(x_{E}(t), x_{I}(t), x_{S}(t), x_{R}(t)\right)$.

Denotemos por $X_{S}$ o conjunto de todos os estados livres da doença; ou seja, o conjunto cujos elementos $x=x(t)$ não possuem indivíduos infectados. Por essa razão, as $m$ primeiras coordenadas de um vetor $x=x(t) \in X_{S}$ são nulas; lembrando que, pela ordenação adotada, essas coordenadas deveriam corresponder às populações de indivíduos infectados, que, no entanto são populações nulas nos estados livres de doença. Isso é:

$$
X_{S}=\left\{x(t)=\left(x_{1}(t), x_{2}(t), \ldots x_{n}(t)\right) \text { tal que } x_{i}(t)=0, i=1, \ldots \ldots, m ; m<n\right\}
$$

É importante distinguir novas infecções de todas as outras mudanças nas populações dos compartimentos. Como exemplo, no modelo SEIR, as novas infecções são somente oriundas do fluxo de indivíduos que deixam de ser suscetíveis e passam a ser expostos (infectados no estágio de latência da doença). Já o fluxo do compartimento de expostos para o compartimento de indivíduos infectantes, não contribui para novas infecções. Esse último fluxo representa uma evolução da doença, sem novos indivíduos infectados.

Sejam $\mathcal{F}_{i}(x)$ a taxa de surgimento de novas infecções no compartimento $i, V_{i}^{+}(x)$ a taxa de transferência de indivíduos para dentro do compartimento $i$ vindo de quaisquer outros compartimentos e $v_{i}^{-}(x)$ a taxa de remoção de indivíduos do compartimento $i$. Devido ao modelo matemático, assumimos que cada função seja, pelo 
menos, de classe $C^{1}$. Ao sistema de compartimentos associamos o sistema de equações diferenciais:

$$
\frac{d x_{i}}{d t}=f_{i}=\mathcal{F}_{i}(x)-v_{i}(x), i=1, \ldots \ldots, n
$$

em que $v_{i}(x)=v_{i}^{-}(x)-\nu_{i}^{+}(x)$ e as funções satisfazem certas premissas, decorrentes da interpretação do modelo epidemiológico.

Designando por:

$$
\mathcal{F}(x)=\left(\mathcal{F}_{1}(x), \mathcal{F}_{2}(x), \ldots, \mathcal{F}_{n}(x)\right) \text { e } \mathcal{V}(x)=\left(\mathcal{V}_{1}(x), \mathcal{V}_{2}(x), \ldots, \mathcal{V}_{n}(x)\right),
$$

escrevemos o sistema acima na forma matricial:

$$
\frac{d x}{d t}=f(x)=\mathcal{F}(x)-\mathcal{V}(x)
$$

sendo $f: D \subset R^{n} \rightarrow R^{n}, f(x)=\left(f_{1}(x), f_{2}(x), \ldots \ldots, f_{n}(x)\right)$ e $f \in C^{1}$.

Definição 2.1 (Ponto de Equilíbrio Livre da Doença) Um ponto de equilíbrio do sistema (2.a) é dito Ponto de Equilíbrio Livre da Doença, aqui denotado por ELD, se ele pertencente ao conjunto $X_{S}$; isto é, se é um estado livre da doença.

Portanto, se $x^{0} \in X_{S}$, esse ponto tem as suas primeiras $m$ coordenadas nulas, sendo da forma: $x^{0}=\left(0, \ldots \ldots 0, x_{m+1}^{0}, \ldots, x_{n}^{0}\right)$.

No modelo considerado, o sistema (2.a) será autônomo, quase-linear; e, de modo geral, poderá ter mais de um ponto de equilíbrio, inclusive do tipo ELD. Mas, supondo que os pontos de equilíbrio do sistema sejam isolados, será realizado um estudo local do modelo matemático, analisado o que ocorre numa vizinhança de um ponto do tipo ELD. Numa vizinhança de um o ponto de equilíbrio isolado do tipo ELD, ele é único. Do ponto de vista da doença, estamos supondo que se uma população de indivíduos está próxima de um ponto de equilíbrio isolado do tipo ELD, então ela permanece próxima a esse ponto de equilíbrio. Isso significa que a introdução, na população, de um número pequeno de indivíduos infectados, não resultará em uma epidemia e a população deverá retornar ao ponto de equilíbrio ELD, uma vez que o número de novas infecções é desprezível.

\section{Premissas do Modelo}

Para que o modelo matemático seja adequado para descrever a transmissão doença, devemos adotar algumas premissas que considerem as condições 
epidemiológicas da doença. Apresentamos, com detalhes, as premissas do modelo ((DRIESSCHE; WATMOUGH, 2002, p. 31-32).

O modelo de transmissão da doença consiste em condições não-negativas e, como as funções $\mathcal{F}_{i}, \mathcal{V}_{i}^{+}, \mathcal{V}_{i}^{-}$representam transferências direcionadas de indivíduos, essas funções são todas não-negativas. Portanto,

(A1) Considerando o vetor $x=x(t) \geq 0$, então $\mathcal{F}_{i}, V_{i}^{+}, v_{i}^{-} \geq 0$ para $i=1, \ldots \ldots, n$.

Se um compartimento é vazio, então não pode ter transferência de indivíduos para fora do compartimento por meio de morte, infecção ou outro meio. Dessa forma, (A2) Se $x_{i}=x_{i}(t)=0$, então $v_{i}^{-}=0$. Isto é, a taxa de remoção de indivíduos do compartimento $i$ é nula. Em particular, se $x=x(t) \in X_{s}$, então $\nu_{i}^{-}=0$ para $i=1, \ldots \ldots, m$.

A incidência de infecção para compartimentos não infectados é zero, assim:

(A3) $\mathcal{F}_{i}=0$ para $i>m$.

No modelo aqui considerado, queremos garantir que o conjunto dos estados livre de doença, $X_{S}$, seja invariante, ou seja, uma vez que a população esteja livre da doença, então a população permanecerá livre da doença; isto é, uma vez que a população tenha adquirido a doença e tenha se recuperado, ela estará imunizada. Portanto, não haverá novas infecções e nem imigração de indivíduos infectados para os primeiros $m$ compartimentos. Essa condição se traduz por:

(A4) Se $x=x(t) \in X_{S}$, então $\mathcal{F}_{i}(x)=0$ e $\nu_{i}^{+}(x)=0$ para $i=1, \ldots \ldots, m$.

As últimas condições dizem respeito ao modelo matemático. São hipóteses sobre a diferenciabilidade da função $f$ do sistema (2.a), para garantir a existência e unicidade de soluções do sistema de equações diferenciais, ao ser fixada uma condição inicial; e, sobre os pontos de equilíbrio do sistema.

Consideremos então o sistema autônomo (2.a), em que a função $f$ seja de classe $C^{1}$; e, suponhamos que esse sistema tenha um ponto de equilíbrio, isolado, do tipo ELD, com as $m$ primeiras coordenadas nulas, $x^{0}=\left(0, \ldots . .0, x_{m+1}^{0}, \ldots x_{n}^{0}\right)$. Em geral o sistema (2.a) não é linear, mas é quase-linear, então podemos considerar o sistema linear ou modelo linearizado dado em (2.b), que aproxima o sistema (2.a), numa vizinhança do ponto de equilíbrio $x^{0}$ :

$$
\frac{d x}{d t}=D f\left(x^{0}\right)\left(x-x_{0}\right)
$$


Em que $D f\left(x^{0}\right)=\left[\frac{\partial f_{i}}{\partial x_{j}}\left(x^{0}\right)\right], 1 \leq i \leq n, 1 \leq j \leq n$, é a matriz Jacobiana da função $f$ em $x^{0}$.

Restringimos nossa atenção para um sistema no qual, numa vizinhança do ponto ELD, há ausência de novas infecções ou a ocorrência de novas infecções pode ser desprezada. Isso nos dá a condição (A5) abaixo:

(A5) Se $\mathcal{F}(x)$ é definido como nulo, então todos os autovalores de $D f\left(x_{0}\right)$ tem parte real negativa

As condições (A1) - (A5), listadas acima, permitem obter uma partição da matriz $D f\left(x_{0}\right)$ em blocos, como nos Lemas 3.1 e Lema 3.2 a seguir. Para a demonstração desses lemas, recordemos que a derivada parcial de uma função $f_{i}: D \subset R^{n} \rightarrow R$, em relação à coordenada $x_{j}$, em um ponto $x^{0}$, é dada por:

$$
\frac{\partial f_{i}}{\partial x_{j}}\left(x^{0}\right)=\lim _{h \rightarrow 0}\left(\frac{f_{i}\left(x^{0}+h e_{j}\right)-f_{i}\left(x^{0}\right)}{h}\right)
$$

sendo $e_{j}$ o j-ésimo vetor da base canônica de $R^{n}$.

Lema 3.1 Se $x^{0}$ é um ponto ELD isolado de (2.a) e a função $f=\mathcal{F}-\mathcal{V}$ satisfaz as condições (A1) - (A4), então a matriz $D \mathcal{F}\left(x_{0}\right)$ tem uma partição em blocos da forma:

$$
D \mathcal{F}\left(x^{0}\right)=\left[\begin{array}{ll}
F & 0 \\
0 & 0
\end{array}\right]
$$

em que $F$ é uma matriz quadrada de ordem $m$, definida por $F=\left[\frac{\partial \mathcal{F}_{i}}{\partial x_{j}}\left(x^{0}\right)\right]$, sendo os índices $1 \leq i, j \leq m$ e $m<n$. Além disso $\mathrm{F}$ é uma matriz tal que todas suas entradas são não-negativas.

\section{Demonstração}

Se $x^{0}$ é um ponto ELD, $x^{0} \in X_{S}$, logo tem as suas $m$ primeiras coordenadas nulas, $m<n$; isto é, $x^{0}=\left(0, \ldots . .0, x_{m+1}^{0}, \ldots x_{n}^{0}\right)$.

(i) Da condição (A3), $\mathcal{F}_{i}=0, i>m$, portanto, $\frac{\partial \mathcal{F}_{i}}{\partial x_{j}}\left(x^{0}\right)=0$, para $i>m$ e para qualquer que seja o índice $j$.

(ii) Seja $e_{j}$ o j-ésimo vetor da base canônica de $R^{n}$ e consideremos a derivada parcial da função coordenada $\mathcal{F}_{i}: D \subset R^{n} \rightarrow R$, em relação à coordenada $x_{j}$, no ponto ELD $x^{0}$ para o índice $j$ tal que $m+1 \leq j \leq n$. Uma vez que $x^{0}$ tem as suas $m$ primeiras coordenadas nulas, por (2.c), a derivada parcial $\frac{\partial F_{i}}{\partial x_{j}}\left(x^{0}\right)$ será dada pelo limite: 


$$
\lim _{h \rightarrow 0}\left(\frac{\mathcal{F}_{i}\left(\left(0, \ldots, 0, x_{m+1}^{0}, \ldots, x_{j}^{0}+h, \ldots, x_{n}^{0}\right)\right)-\mathcal{F}_{i}\left(0, \ldots, 0, x_{m+1}^{0}, \ldots, x_{j}^{0}, \ldots, x_{n}^{0}\right)}{h}\right)
$$

Mas, da condição $(\mathrm{A} 4), \mathcal{F}_{i}\left(x^{0}\right)=0$, para $1 \leq i \leq m$. Logo, $\frac{\partial \mathcal{F}_{i}}{\partial x_{j}}\left(x^{0}\right)=0$, para $1 \leq i \leq m$ e $m+1 \leq j \leq n$.

(iii) Por (2.c) e (A4), para as derivadas parciais $\frac{\partial F_{i}}{\partial x_{j}}\left(x^{0}\right)$ para os índices $1 \leq i \leq m \mathrm{e}$ $1 \leq j \leq m$, temos:

$$
\begin{gathered}
\lim _{h \rightarrow 0^{+}}\left(\frac{\mathcal{F}_{i}\left(\left(0, \ldots, 0, h, 0 \ldots, 0, x_{m+1}^{0}, \ldots, x_{n}^{0}\right)\right)-\mathcal{F}_{i}\left(0, \ldots \ldots, 0, x_{m+1}^{0}, \ldots, x_{j}^{0}, \ldots, x_{n}^{0}\right)}{h}\right) \\
\frac{\partial \mathcal{F}_{i}}{\partial x_{j}}\left(x^{0}\right)=\lim _{h \rightarrow h^{+}} \frac{\mathcal{F}_{i}\left(0, \ldots, h_{m, 0,} x_{m+1}^{0}, \ldots, x_{n}^{0}\right)}{h}
\end{gathered}
$$

Nesse caso, o limite deve ser somente à direita devido ao domínio da função, pois cada coordenada do ponto $x^{0}$ representa uma população de indivíduos.

De (2.e) e (A1), as derivadas parciais $\frac{\partial F_{i}}{\partial x_{j}}\left(x^{0}\right) \geq 0$, para os índices $1 \leq i \leq m \mathrm{e}$ $1 \leq j \leq m$.

Segue dos itens (i) e (ii):

$$
D \mathcal{F}\left(x^{0}\right)=\left[\begin{array}{ll}
F & 0 \\
0 & 0
\end{array}\right]
$$

com $F=\left[\frac{\partial \mathcal{F}_{i}}{\partial x_{j}}\left(x^{0}\right)\right], \quad 1 \leq i, j \leq m$ e $m<n$. Do item (iii), temos que a matriz $F$ tem todas as suas entradas não negativas.

Lema 3.2 Se $x^{0}$ é um ponto ELD isolado de (2.a) e a função $f=\mathcal{F}-\mathcal{V}$ satisfaz as condições (A1) - (A5), então a matriz $D \mathcal{V}\left(x^{0}\right)$ tem uma partição em blocos da forma:

$$
D V\left(x^{0}\right)=\left[\begin{array}{cc}
V & 0 \\
U & W
\end{array}\right]
$$

em que $V$ é uma matriz quadrada de ordem $m$, definida por $V=\left[\frac{\partial v_{i}}{\partial x_{j}}\left(x^{0}\right)\right]$ sendo os índices $1 \leq i_{n} j \leq m$ e $m<n$. Além disso, $V$ é invertível e todos os autovalores das matrizes $V$ e $W$ têm parte real positiva.

\section{Demonstração}

Seja $x^{0}$ um ponto ELD, isolado. Como $x^{0} \in X_{S}, x^{0}=\left(0, \ldots . .0, x_{m+1}^{0}, \ldots x_{n}^{0}\right)$.

(i) Da expressão da matriz jacobiana, por (A2) e (A4), temos que: 
$v\left(x^{0}\right)=v_{i}^{-}\left(x^{0}\right)-v_{i}^{+}\left(x^{0}\right)=0,1 \leq i \leq m$ e $m+1 \leq j \leq n$.

Logo, as derivadas parciais $\frac{\partial v_{i}}{\partial x_{j}}\left(x_{0}\right)=0$, para $1 \leq i \leq m$ e $m+1 \leq j \leq n$.

(ii) $\quad$ Seja $1 \leq i \leq m$ e $1 \leq j \leq m$, com $i \neq j$. Sendo $i \neq j$, a i-ésima coordenada do vetor $x^{0}+h e_{j}$ é nula e por (A2), $\nu_{i}^{-}=0$. Por (A4), para $x^{0} \in X_{S}, V_{i}^{+}=0$ para $1 \leq i \leq m$. Assim, a derivada parcial é dada pelo limite:

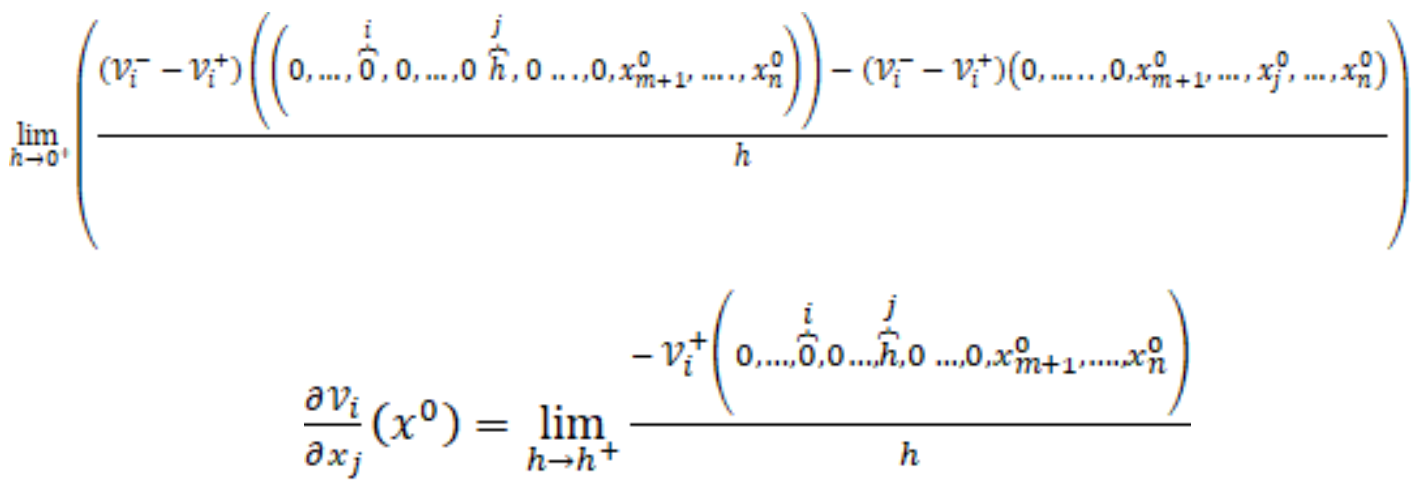

Da condição (A1) e (2.f), $\frac{\partial v_{i}}{\partial x_{j}}\left(x^{0}\right) \leq 0$, para $1 \leq i \leq m$ e $1 \leq j \leq m, i \neq j$.

Observamos que para $i=j$ não é possível garantir que $v_{i}^{-}$seja nulo. Assim, o sinal da derivada parcial $\frac{\partial v_{i}}{\partial x_{i}}\left(x^{0}\right)$ dependerá dos fluxos de entrada e saída no compartimento $i$.

Concluímos que a matriz $V=\left[\frac{\partial v_{i}}{\partial x_{j}}\left(x_{0}\right)\right]$, para $1 \leq i, j \leq m$, tem suas entradas, com exceção das que estão na diagonal, menores ou iguais a zero.

(iii) A fim de mostrarmos que a matriz $V$ tem inversa, notemos que todos os autovalores dessa matriz têm parte real positiva. De fato, pela condição (A5), estamos supondo que, uma vez que estejamos em uma vizinhança de um ponto de equilíbrio já livre da doença, não haverá reinfecção (novas infecções). Nessa situação, consideramos $F$ nulo e a dinâmica do sistema (2.b), numa vizinhança do ponto de equilíbrio $x^{0}$, é dada pelo sistema linear:

$$
\frac{d x}{d t}=-D \mathcal{V}\left(x^{0}\right)\left(x-x^{0}\right)=-\left[\begin{array}{cc}
V & 0 \\
U & W
\end{array}\right]\left[x-x^{0}\right]
$$

Ainda por (A5), os autovalores da matriz $D(-\mathcal{V})\left(x^{0}\right)$ têm parte real negativa. Como a matriz é dada em blocos, os autovalores da matriz $D(-\mathcal{V})\left(x^{0}\right)$ são os autovalores da matriz $-V$ e os autovalores da matriz $-W$. Portanto as matrizes $V$ e $W$ têm todos os autovalores com parte real positiva. Como consequência, as matrizes $V$ e $W$ são invertíveis. 
Portanto, do ponto de vista da análise qualitativa do sistema autônomo (2.a), as suas soluções se comportam, numa vizinhança de um ponto ELD, como as soluções do sistema linear, obtido de (2.d) e (2.f):

$$
D f\left(x^{0}\right)=\left[\begin{array}{cc}
F-V & 0 \\
-U & -W
\end{array}\right]\left[x-x^{0}\right]
$$

\section{A Matriz Próxima Geração e sua Interpretação Epidemiológica}

Nosso intuito é determinar o Número Básico de Reprodução da doença, $\mathrm{R}_{0}$, a partir do sistema (2g). Para isso, iremos apresentar um método introduzido por Diekmann, Heesterbeek e Metz (1990), em que esse número é obtido através de um operador chamado de Next Generation Operator. Para o caso em que o modelo matemático é um sistema de equações diferenciais, esse operador é uma matriz, chamada de Matriz Próxima Geração, aqui denotada por MPG, formada por blocos das matrizes (2.d) e (2.f), conforme a definição dada abaixo.

Definição 4.1 (Matriz Próxima Geração) A Matriz Próxima Geração, denotada por MPG, é a matriz quadrada de ordem $m, m<n$, dada por $F V^{-1}$.

Para a justificativa da fórmula da MPG, consideremos o vetor $\psi(0)=\left(\psi_{1}(0), \ldots, \psi_{m}(0)\right)$, tal que cada coordenada é o número inicial de indivíduos infectados no compartimento $i$ para $1 \leq i \leq m$ e $\quad$ seja $\psi(t)=\left(\psi_{1}(t), \ldots, \psi_{m}(t)\right), m<n$, o vetor representando o número desses indivíduos, inicialmente infectados, que permanecem nos compartimentos infectados após $t$ unidades de tempo. Lembremos que os compartimentos correspondentes às populações de indivíduos infectados são as $m$ primeiras coordenadas do vetor $x=x(t)=\left(x_{1}, x_{2}, \ldots x_{n}\right)$, que representa a população de indivíduos em cada um dos compartimentos. O sistema (2.g) que tem uma única solução $\psi(t)=e^{-V t} \psi(0)$ onde a exponencial de uma matriz A quadrada é definida como sendo o operador ou matriz $e^{A}=\sum_{k=0}^{+\infty} \frac{A^{k}}{k !}$, que é absolutamente convergente para qualquer matriz $A$ e cujo primeiro termo é a matriz identidade I (HIRSCH; SMALE, 1974, 83).

Ao se fazer a modelagem matemática de um problema real, em geral, só dispomos de dados experimentais da população ou de uma amostra da população. Dessa 
forma, usando conceitos de Estatística (HOFFMANN; BRADLEY, 1999, p.330-334). Consideramos o vetor $\psi=\psi(t)$ como uma variável aleatória contínua. Cada a entrada $(i, j)$ da matriz $F$ pode ser vista como uma função densidade de probabilidade para novas infecções no compartimento $i$, ocasionadas por um único indivíduo infectado do compartimento $j$. O vetor $E$, cujas coordenadas exprimem as médias ou valores esperados de novas infecções nos compartimentos $i, 1 \leq i \leq m$, causadas pelos indivíduos inicialmente infectados será dado pela integral imprópria:

$$
\begin{gathered}
E=\int_{0}^{+\infty} F \psi(t) d t \\
\int_{0}^{+\infty} F \psi(t) d t=F \int_{0}^{+\infty} e^{-V t} \psi(0) d t=F\left[\lim _{s \rightarrow+\infty}-V^{-1} e^{-V t}+V^{-1} I\right] \psi(0)= \\
F\left[\lim _{s \rightarrow+\infty}-V^{-1} e^{-V t} \psi(0)\right]+F V^{-1} I \psi(0)=F\left[\lim _{s \rightarrow+\infty}-V^{-1} \psi(s)\right]+F V^{-1} \psi(0)
\end{gathered}
$$

Pelo Lema 2.2, a matriz $V$ é invertível e todos os seus autovalores tem parte real positiva, assim os autovalores da matriz $-V$ têm parte real negativa. Como $\psi(t)=e^{-V s} \psi(0)$, temos que $\lim _{\Omega \rightarrow+\infty} \psi(s)=0$ (HIRSCH; SMALE, 1974, p. 136). Portanto:

$$
E=\int_{0}^{+\infty} F \psi(t) d t=F V^{-1} \psi(0)
$$

Do ponto de vista de Epidemiologia, a função $v_{j}(x)=v_{j}^{-}(x)-v_{j}^{+}(x)$ representa os fluxos de saída e entrada de indivíduos, no compartimento $j$ devidos a qualquer motivo que não sejam novas infecções. Dessa forma a entrada $(j, k)$ de $V^{-1}$ representa o período médio de tempo que um indivíduo permanece no compartimento $j$ durante a sua vida útil, assumindo que a população permanece próximo de DFE e impedindo reinfecção. Consequentemente, a entrada $(i, k)$ da matriz produto $F V^{-1}$ é o número esperado para novas infecções no compartimento $i$, produzidas por indivíduo infectado que foi introduzido originalmente no compartimento $k$, assumindo que a população do compartimento $i$ é inteiramente suscetível à doença.

\section{Matriz Próxima Geração e o Número Básico de Reprodução da Doença}

A matriz $\mathrm{FV}^{-1}$, tem algumas características especiais que serão importantes para o estudo da dinâmica da doença. Dentre elas, a MPG é uma matriz quadrada com todas 
as suas entradas não-negativas. Outra propriedade fundamental da MPG é o fato dela ter um auto valor real positivo dominante e qualquer outro possível autovalor da MPG, real ou complexo, tem seu módulo estritamente menor ou igual a esse auto valor dominante. As propriedades detalhadas da MPG podem ser consultadas em (SILVA, 2017, p. 3540).

Definição 5.1 (Raio Espectral de uma Matriz) Seja $A=\left[a_{i j}\right]$ uma matriz real quadrada de ordem $n$. O raio espectral de $A$ é o número real $\rho(A)$, que é o máximo dentre os módulos dos autovalores da matriz. Isto é,

$$
\rho(A)=\max \{|\lambda|, \lambda \text { um auto valor de } A\} \text {. }
$$

Assim, $\rho\left(F V^{-1}\right)$ é um autovalor da matriz $F V^{-1}$ e todos os autos autovalores dessa matriz têm módulo menor ou igual a $\rho\left(F V^{-1}\right)$.

Considerando $x^{0}$ um ponto de equilíbrio livre da doença, ELD, do sistema que modela a transmissão da doença e $F V^{-1}$ a MPG a ele associada, é provado em (DRIESSCHE; WATMOUGH, 2002, p. 33-34) que:

(i) se $\rho\left(F V^{-1}\right)<1$, então $x^{0}$ é um ponto de equilíbrio localmente assintoticamente estável, o que significa que, numa vizinhança do ponto de equilíbrio $x^{0}$, todas as soluções do sistema autônomo convergem para $x^{0}$, que é um ponto livre de doença.

(ii) se $\rho\left(F V^{-1)}>1\right.$, então $x^{0}$ é um ponto de equilíbrio instável.

Por outro lado, segundo Driessche e Watmough (2002, p. 30):

(iii) se $R_{0}<1$, a doença tenderá a ser eliminada ou poderá ser mantida em níveis aceitáveis.

(iv)se $R_{0}>1$, haverá um surto epidêmico.

Desta forma o $R_{o}$ é um indicador do limiar epidêmico da doença e a sua determinação permite a compreensão da dinâmica da propagação da doença.

Das condições (i), (ii), (iii) e (iv), estabelecemos a relação entre MPG e o número $R_{0}<1$.

Definição 5.2 (Relação entre MPG e $\boldsymbol{R}_{0}$ ) Definimos o Número Básico de Reprodução da doença $R_{0}$ como sendo o raio espectral da matriz $\mathrm{FV}^{-1}$; isto é:

$$
R_{0}=\rho\left(F V^{-1}\right)
$$




\section{Cálculo Número $R_{0}$ da Leishmaniose Visceral Canina por meio da MPG}

A Leishmaniose visceral, ou calaza, é uma doença transmitida pela fêmea do mosquito-palha ou birigui, um mosquito de coloração palha, cuja espécie é chamada de Lutzomyia longipalpis. Se a fêmea do mosquito (vetor) estiver infectada, ao picar o hospedeiro (homem ou o animal), introduz na circulação do mesmo o protozoário do gênero Leishmania, que é o agente patogênico ou agente infeccioso.

Leishmaniose Visceral, tradicionalmente uma doença rural da América Tropical, do Mediterrâneo, Leste da África, Oriente Médio e China, vem se tornando mais comum em áreas urbanas e suburbanas, por isso é de grande importância aprofundar estudos para obter formas de controle eficiente da doença.

Nos centros urbanos a transmissão se torna potencialmente perigosa por causa da grande quantidade de cachorros, que adquirem a infecção e desenvolvem um quadro clínico semelhante ao do homem. Os cães são considerados os maiores reservatórios da doença nos centros urbanos.

O modelo aqui apresentado tem como referência o apresentado em Dye (1966) e é análogo ao modelo SEIR. As hipóteses do modelo são as seguintes:

- O sistema envolve uma população total de cães, denotada por $D$, que será considerada constante e dividida em 4 categorias de subpopulações ou em 4 compartimentos: $S=S(t)$ cães suscetíveis, $L=L(t)$ cães expostos ou no estado latente, $I=I(t)$ cães infectantes e $R=R(t)$ cães resistentes à doença.

- Dessa forma, $D=S(t)+R(t)+L(t)+I(t)$.

- Todos os cães nascem suscetíveis ou resistentes à doença.

- A taxa de natalidade dos cães é igual à taxa de mortalidade dos mesmos; e portanto, a população total dos cachorros permanece sempre constante, igual a $\mathrm{D}$, ao longo do tempo.

Consideremos o vetor $x=x(t)=(L(t), I(t), S(t), R(t))$, com as primeiras coordenadas sendo as populações de indivíduos infectados (latentes e infectantes) e o sistema de equações diferenciais (6.a) apresentado por Dye (1966, p.126). O sistema (6.a) é autônomo quase-linear. 


$$
\left\{\begin{array}{l}
\frac{d L}{d t}=C_{d} I \frac{S}{D}-\left(\theta_{d}+\delta_{d}\right) L \\
\frac{d I}{d t}=\theta_{d} L-\delta_{d} I \\
\frac{d S}{d t}=\alpha_{d} \beta_{d} D-C_{d} I \frac{S}{D}-\delta_{d} S \\
\frac{d R}{d t}=\left(1-\alpha_{d}\right) \beta_{d} D-\delta_{d} R
\end{array}\right.
$$

Fonte: DYE, 1966, p. 127

onde: $\mathrm{D}, C_{d}, \alpha_{d}, \beta_{d}, \delta_{d}, \theta_{d}$ são constantes positivas:

D - População total de cães;

$C_{d^{-}}$Capacidade vetorial da população de mosquitos fêmea de transmitir infecção entre cães ;

$\alpha_{d}$ Proporção de cães que nascem suscetíveis à doença;

$\beta_{d}-$ Taxa de nascimento dos cães;

$\delta_{d}$ - Taxa de mortalidade dos cães;

$\theta_{d}$ - Taxa segundo a qual cães infectados, na fase latente, tornam-se infectantes;

$t=\frac{1}{\theta_{d}}$ - é o período de incubação da doença.

Nesse modelo, a taxa de mortalidade de cães, $\delta_{d}$, não considera as mortes causadas por sacrifício do animal. As mortes consideradas são as mortes devidas à velhice, doenças ou outra causa natural.

O ponto de equilibro, não trivial, do sistema (6.a) que é do tipo ELD (livre da doença), é $P_{E}=\left(0,0, S_{E}, R_{E}\right)=\left(0,0, \alpha_{d} D,\left(1-\alpha_{d}\right) D\right)$, pois há ausência de populações infectadas. As variáveis $S_{E}$ e $R_{E}$ são obtidas igualando as duas últimas equações do sistema (6.a) a zero e usando a hipótese de que os cães nascem suscetíveis ou resistentes à doença. Como o sistema (6.a) é quase-linear, é possível aproximá-lo, numa vizinhança do ponto de equilíbrio $P_{E}$, a um sistema linear cuja a matriz é a matriz jacobiana no ponto de equilíbrio.

As novas infecções são decorrentes apenas do fluxo que sai do compartimento dos cães suscetíveis para o compartimento dos cães expostos. $\mathrm{O}$ fluxo de cães do compartimento dos expostos para o compartimento dos infectantes não resulta em novas infecções. Esse fluxo representa apenas uma evolução da doença, mas não aumenta o número de indivíduos infectados. Desta forma, o sistema (6.a) pode ser reescrito sob a forma matricial: 


$$
\left[\begin{array}{l}
\frac{d L}{d t} \\
\frac{d I}{d t} \\
\frac{d S}{d t} \\
\frac{d R}{d t}
\end{array}\right]=\mathcal{F}(x)-v(x)=\left[\begin{array}{c}
C_{d} I \frac{S}{D} \\
0 \\
0 \\
0
\end{array}\right]-\left[\begin{array}{c}
\left(\theta_{d}+\delta_{d}\right) L \\
-\theta_{d} L+\delta_{d} I \\
-\alpha_{d} \beta_{d} D+C_{d d} I \frac{S}{D}+\delta_{d} S \\
-\left(1-\alpha_{d}\right) \beta_{d} D+\delta_{d} R
\end{array}\right]
$$

De (6.b), a matriz Jacobiana de $\mathcal{F}=\mathcal{F}(x)$, avaliada no ponto de equilíbrio livre da doença, ELD, $P_{E}=\left(0,0, S_{E}, R_{E}\right)=\left(0,0, \alpha_{d} D,\left(1-\alpha_{d}\right) D\right)$, será:

$$
D \mathcal{F}\left(P_{E}\right)=\left[\begin{array}{ccccc}
0 & \frac{C_{d}}{D} S_{E} & 0 & 0 \\
0 & 0 & 0 & 0 \\
0 & 0 & 0 & 0 \\
0 & 0 & 0 & 0
\end{array}\right]=\left[\begin{array}{cccc}
0 & C_{d} \alpha_{d} & 0 & 0 \\
0 & 0 & 0 & 0 \\
0 & 0 & 0 & 0 \\
0 & 0 & 0 & 0
\end{array}\right]=\left[\begin{array}{ll}
F & 0 \\
0 & 0
\end{array}\right]
$$

sendo o bloco $F$ uma matriz quadrada de ordem 2, que corresponde a populações de cães infectados.

$$
F=\left[\begin{array}{cc}
0 & C_{d} \alpha_{d} \\
0 & 0
\end{array}\right]
$$

Analogamente, de (6.b), a matriz jacobiana da função $v=V(x)$ avaliada no ponto ELD, $P_{E}=\left(0,0, S_{E}, R_{E}\right)=\left(0,0, \alpha_{d} D,\left(1-\alpha_{d}\right) D\right)$, será:

$$
D V\left(P_{E}\right)=\left[\begin{array}{cccc}
\left(\theta_{d}+\delta_{d}\right) & 0 & 0 & 0 \\
-\theta_{d} & \delta_{d} & 0 & 0 \\
0 & \frac{S_{E}}{D} & \delta_{d} & 0 \\
0 & 0 & 0 & \delta_{d}
\end{array}\right]=\left[\begin{array}{cccc}
\left(\theta_{d}+\delta_{d}\right) & 0 & 0 & 0 \\
-\theta_{d} & \delta_{d} & 0 & 0 \\
0 & \frac{S_{E}}{D} & \delta_{d d} & 0 \\
0 & 0 & 0 & \delta_{d}
\end{array}\right]=\left[\begin{array}{cc}
V & 0 \\
U & W
\end{array}\right]
$$

sendo o bloco $V$ e a sua inversa $V^{-1}$ dadas por:

$$
V=\left[\begin{array}{cc}
\theta_{d}+\delta_{d} & 0 \\
-\theta_{d} & \delta_{d d}
\end{array}\right], V^{-1}=\frac{1}{\left(\theta_{d}+\delta_{d}\right) \delta_{d d}}\left[\begin{array}{cc}
\delta_{d d} & 0 \\
\theta_{d d} & \theta_{d d}+\delta_{d}
\end{array}\right]=\left[\begin{array}{cc}
\frac{1}{\theta_{d}+\delta_{d d}} & 0 \\
\frac{\theta_{d d}}{\left(\theta_{d}+\delta_{d}\right) \delta_{d d}} & \frac{1}{\delta_{d d}}
\end{array}\right]
$$

A MPG, conforme já visto, será $\mathrm{G}=F V^{-1}$. Portanto, de (6.c) e (6.d), temos:

$$
G=F V^{-1}=\left[\begin{array}{cc}
\frac{c_{d} \alpha_{d} \theta_{d}}{\left(\theta_{d}+\delta_{d}\right) \delta_{d d}} & \frac{c_{d d} \alpha_{d}}{\delta_{d}} \\
0 & 0
\end{array}\right]
$$

O Número Básico de Reprodução da Leishmaniose Visceral Canina $R_{0}$ é o raio espectral da matriz $G$ dada em (6.e), lembrando que o raio espectral de uma matriz é o autovalor dominante isto é, o autovalor que tem o maior módulo dentre todos os autovalores da matriz. Os autovalores da matriz (6.e) são $\mu=0$ e $\lambda=\frac{c_{d} \alpha_{d} \theta_{d}}{\left(\theta_{d}+\delta_{d}\right) \delta_{d}}$. Como o raio espectral da matriz é o autovalor não-nulo, temos que: 


$$
R_{0}=\lambda=\frac{c_{d} \alpha_{d} \theta_{d}}{\left(\theta_{d}+\delta_{d}\right) \delta_{d}}
$$

Este número $R_{0}$ é precisamente o citado por Dye (1966, p. 126).

\section{Conclusão}

A funcionalidade do método que utiliza a Matriz Próxima Geração (MPG) foi verificada, através da sua aplicação no estudo da dinâmica da Leishmaniose Visceral Canina. A MPG permite determinar o $\mathrm{R}_{0}$ de forma simples e imediata, através do cálculo dos seus autovalores. O método também pode ser aplicado para a determinação de outras doenças infecciosas que possam se modeladas por um sistema autônomo de equações diferenciais. Portanto, é um procedimento que pode ser adotado, de modo geral, em estudos epidemiológicos para os quais um sistema autônomo seja adequado.

Uma contribuição desse trabalho foi o de apresentar os cálculos para a determinação do $R_{0}$ para a Leishmaniose Canina por meio do método da MPG, preenchendo os detalhes omitidos por Dye (1966), bem como detalhar resultados apresentados nas outras referências citadas.

\section{Referências}

DIEKMANN, O.; HEESTERBEEK, J. A. P.; METZ, J. A. J.: On the definition and the computation of the basic reproduction ratio $\mathrm{R}_{0}$ in models for infectious diseases in heterogeneous populations. Journal of Mathematical Biology, 28 (4) (1990) 365-382.

DRIESSCHE P. van den; WATMOUGH, J.: Reproduction numbers and sub-threshold endemic equilibria for compartmental models of disease transmission. Mathematical Biosciences 180 (2002), 29-48.

DYE, C.: The Logic of Visceral Leishmaniasis Control. The American Journal of Tropical Medicine and Hygiene, vol. 55 (1966), 125-130.

HIRSCH, M. W.; SMALE, S.: Differential Equations, Dynamical Systems, And Linear Algebra. New York, San Francisco, London: Academic Press, 1974.

HOFFMAnN, L. D.; BRAdLeY, G. L.: Cálculo Um Curso Moderno E Suas Aplicações. $6^{a}$ ed., Rio de Janeiro: LTC-Livros Técnicos e Científicos, 1999.

SILVA, Rômulo Fernando Domingos da: Modelagem Matemática da Leishmaniose Visceral. 57 f. Trabalho de Conclusão de Curso (Bacharelado em matemática) Instituto de Matemática e Estatística, Universidade do Estado do Rio de Janeiro, Rio de Janeiro, 2017. 